\title{
Las medusas Scyphozoa de la Bahía Blanca, Argentina*
}

\author{
Hermes W. MIANZAN**
}

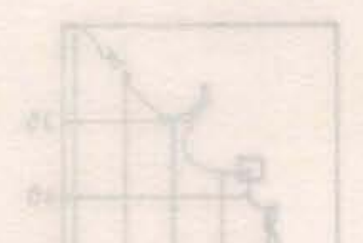

Instituto Nacional de Investigación y Desarrollo Pesquero

(CC 175, 7600 Mar del Plata, Argentina)

\begin{abstract}
- Abstract: Juvenils and adults of Chrysaora lactea, Aurelia aurita and Drymonema gorgo were found in Blanca Bay during summer and efirae of the two first occurred from October to January. These species are commonly found in Brazilian waters. Their occurrence in higher latitudes may be either due to the influence of warm waters coming from north or to the heating of local waters which could maintain their populations temporarily during spring and summer. In this period C. lactea and $A$. aurita may reproduce as it is suggested by the occurrence of their efirae in the surveyed area.
\end{abstract}

- Descriptors: Scyphozoa, Medusae, Chrysaora lactea, Aurelia aurita, Drymonema gorgo, Temporal distribution, Abundance, Zoogeography, Bahía Blanca: Argentina.

- Descritores: Scyphozoa, Medusae, Chrysaora lactea, Aurelia aurita, Drymonema gorgo, Distribuição temporal, Abundância, Zoogeografia, Bahía Blanca: Argentina.

\section{Introduccíon}

Tres especies de cifomedusas han sido halladas en las aguas de Bahía Blanca $\left(38^{\circ} 30^{\prime}\right.$ - $39^{\circ} 25^{\prime}$ 'S y $61^{\circ} 30^{\prime}$ $62^{\circ} 20^{\circ} \mathrm{W}$ ); Chrysaora lactea Eschscholz, 1829 (Vannucci \& Tundisi, 1962), Drymonema gorgo Muller, 1883 y Aurelia aurita (Linné, 1758) (Mianzan, 1986). Las dos primeras son endémicas del océano Atlántico sudoccidental (Vannucci \& Tundisi, op. cit.). A. aurita en cambio es cosmopolita (Kramp, 1961; Russell, 1970; Möller, 1980) y común en la región subantártica norte entre las isotermas de 10 y $20^{\circ} \mathrm{C}$ (Larson, 1986).

El objetivo del trabajo es aportar información sobre la distribución espacial, temporal y abundancia de los diferentes estados de desarrollo de las tres especies citadas, encontrados en el plancton de Bahía Blanca.

\section{Material y metodos}

Las campañas se realizaron con frecuencia mensual y el detalle de su duración, sector, red utilizada, etc., puede

(*) Contribución Cientifica $\mathrm{n}^{2} 144$ del Instituto Argentino de Oceanografía. Av. Alem 53 - 8000 Bahía Blanca. Argentina.

(**) Becario del Consejo Nacional de Investigaciones Cientificas y Técnicas, CONICET. observarse en la Tabla 1. En cada estación (Fig. 1) se midieron la salinidad y temperatura en superficie. Las efiras se capturaron con red tipo Hensen y los juveniles y adultos con red tipo IKMT (esta red solo se utilizó en 4 estaciones por lo que los datos de abundancia son parciales). Las características físicas de la Bahía Blanca pueden consultarse en los trabajos de Arango (1985), Mianzan \& Sabatini (1985) y Mianzan (1986). Para el análisis taxonómico se utilizaron los trabajos de Mayer (1910), Kramp (1961) y Larson (1986).

Tabla 1. Campañas realizadas en la Bahía Blanca

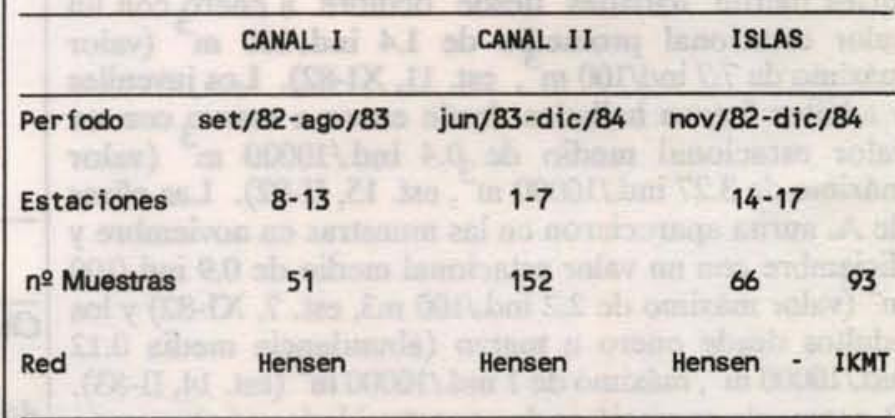




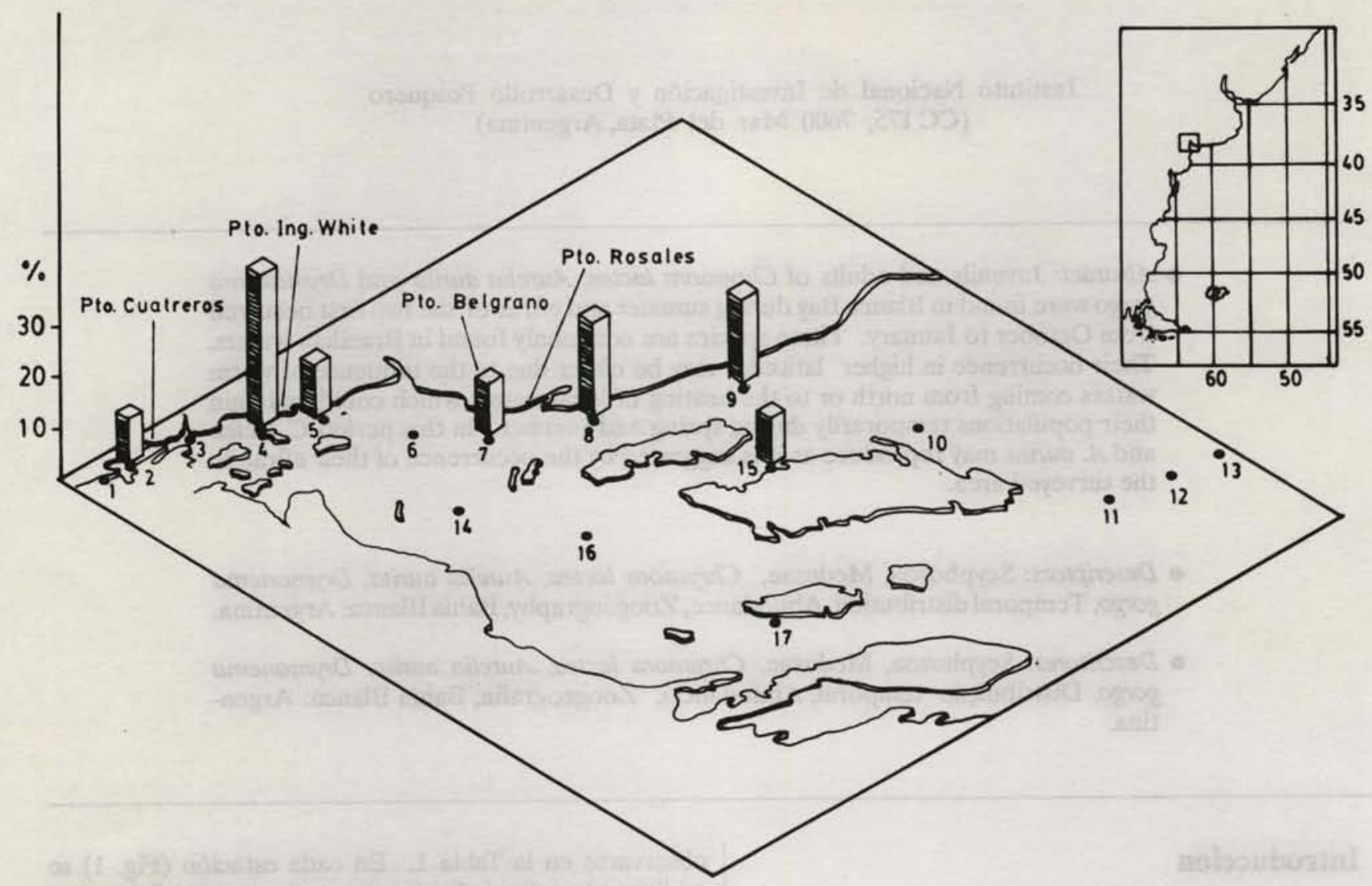

Fig. 1. Ubicación de las estaciones de muestreo y distribución de la frecuencia (\%) de captura de efiras de Chrysaora lactea y Aurelia aurita en la Bahla Blanca (Pcia. de Buenos Aires, Argentina).

\section{Resultados}

C. lactea, A. aurita y D. gorgo aparecieron fundamentalmente durante primavera y verano. Las efiras de $C$. lactea fueron halladas desde octubre a energ, con un valor estacional promedio de 1.4 ind $/ 100 \mathrm{~m}^{3}$ (valor máximo de 7.7 ind $/ 100 \mathrm{~m}^{3}$, est. $11, \mathrm{XI}-82$ ). Los juveniles y adultos fueron hallados desde enero a marzo con un valor estacional medio de 0.4 ind $/ 10000 \mathrm{~m}^{3}$ (valor máximo de 3.27 ind. $/ 10000 \mathrm{~m}^{3}$, est. 15, II-82). Las efiras de A. aurita aparecieron en las muestras en noviembre y diçiembre con un valor estacional medio de 0.9 ind./100 $\mathrm{m}^{3}$ (valor máximo de 2.2 ind./100 m3, est. 7, XI-82) y los adultos desde enero a marzo (abundancia media 0.12 ind. $/ 10000 \mathrm{~m}^{3}$, máximo de 1 ind. $/ 10000 \mathrm{~m}^{3}$ (est. 14, II-83). D. gorgo solo apareció en dos oportunidades y únicamente individuos adultos.

Las tres especies fueron halladas principalmente por fuera de la estación 4 y las efiras, en particular, en estaciones cercanas a zonas portuarias: Pto. Cuatreros (est. 2), Pto. Ing. White (est. 4), Pto. Belgrano (est. 7) y Pto. Rosales (est. 8) (Fig. 1). Los pocos ejemplares capturados en las estaciones más internas (est. 2) coincidieron con condiciones de pleamar, con salinidades próximas a $30 \% 0$ (Tabla 2).

Tabla 2. Períodos de aparición y valores de salinidad y temperatura del agua correspondientes a los hallazgos de efiras y medusas de clfomedusas en Bahia Blanca (1982-1984)

\begin{tabular}{lcccc}
\hline & EFIRAS & MEDUSAS & S\%० & $T^{\circ} \mathrm{C}$ \\
\hline Chrysaora lactea & oct-ene & ene-mar & $28,1-37,9$ & $14,2-23,8$ \\
Aurelia aurita & nov-dic ene-may & $30,6-36,2$ & $14,9-23,2$ \\
Drymonema gorgo & - & feb-mar & - & $19,3-20,4$
\end{tabular}




\section{Discusión y conclusiones}

Las tres especies citadas son comunes a las aguas brasileras (Vannucci \& Tundisi, 1962; Mianzan, 1986) y es posible que algunos ejemplares puedan llegar a tan altas latitudes arrastradas por la deriva templado-cálida (Balech, 1949; 1954), o también que el aumento de temperatura del agua durante la época estival posibilite el desarrollo de poblaciones locales. Los relevamientos bentónicos realizados en el área, tanto de sustrato duro como móvil (Valentinuzzi de Santos, 1971; Martinez, 1982; Elias, 1985; Bremec, 1986) no evidenciaron la presencia de pólipos de Scyphomedusae, sin embargo el hallazgo de efiras recién liberadas nos sugiere que este sector costero reune las condiciones necesarias para la reproducción de estas especies. La distribución de capturas de efiras, principalmente en zonas portuarias donde sería factible el asentamiento de pólipos, respaldaría esta última hipótesis. Para confirmarlo se hace necesario un estudio de madurez gonadal.

Se cita por primera vez el hallazgo simultáneo de efiras, juveniles y adultos de $C$. lactea y $A$. curita en aguas costeras del Atlántico suroeste.

Las cifomedusas no fueron dominantes durante el período muestreado. Su número fue bajo si lo comparamos con las capturas del ctenóforo Mnemiopsis maccradyi Mayer, 1900 y la hidromedusa Olindias sambaquiensis Müller, 1861 recolectados en Bahía Blanca por el mismo procedimiento (Mianzan \& Sabatini, 1985; Mianzan, 1986).

\section{Summary}

This paper deals with preliminar information about species composition, distribution and abundance of Scyphomedusae from Blanca Bay (Argentina). Samples were collected with Hensen and IKMT nets. Three species were found: Chrysaora lactea, Aurelia aurita and Drymonema gorgo in summer, and efirae of the two former from October to January. These species are commonly found in Brazilian waters. Their occurrence in higher latitudes may be due to the influence of warm waters coming from north or the heating of local waters which could maintain their populations temporarily during spring and summer. In this period $C$. lactea and $A$. curita may reproduce as it is suggested by the occurrence of their efirae in the surveyed area.

\section{Agradecimientos}

Agradezco el apoyo material y personal recibido durante mi estadía en el Instituto Argentino de Oceanografía (IADO) y a la Prefectura Naval Argentina. Agradezco además a los Dres. F. Ramirez (INIDEP), C. Bremec (INIDEP) y R. Elias (UNMdP) por su permanente colaboración y estímulo. À la Dra. P. Cervellini(CRUB) por el material de la campaña CANAL II. A los Sres. D. Echenique, C. Bernardez, A. Echevarria y O. Carretero y a los Dres. C. Bremec y R. Elias por su inapreciable ayuda en las tareas de campo.

\section{Referencias bibliográficas}

ARANGO, J. M. 1985. Distribución de parámetros oceanográficos en el estuario de Bahía Blanca. Seminario de Licenciatura en Oceanografía. Universidad Nacional del Sur, Departamento de Física. 46p.

BALECH, E. 1949. Estudio crítico de las corrientes marinas del litoral argentino. Physis, 20(57):159-164.

1954. División zoogeográfica del litoral sudamericano. Revta Biol. mar., Valparaíso, 4(1/3):184-195.

BREMEC, C. 1986. Asociaciones del macrobentos infralitoral de Monte Hermoso (39 $00^{\prime} \mathrm{S}-61^{\circ} 17 \mathrm{~W}$, República Argentina). Spheniscus, 2:1-18.

ELIAS, R. 1985. Macrobentos del estuario de la Bahía Blanca (Argentina). I. Mesolitoral. Spheniscus, 1:1-33.

KRAMP, P. L. 1961. Synopsis of the Medusae of the world. J. mar. biol. Ass. U.K., 40:1-469.

LARSON, R. 1986. Pelagic Scyphomedusae (Scyphozoa: Coronatae and Semaeostomeae) of the Southern Ocean. Biology of the Antarctic Sea XVI. Antarct. Res. Ser., Am. geophys. Un., 41(3):52-165.

MARTINEZ, D. 1982. Estudio sobre las comunidades incrustantes (fouling) de Puerto Ingeniero White. Seminario de Licenciatura. Universidad Nacional del Sur, Departamento de Biologia. 69p.

MAYER, D. G. 1910. Medusae of the world. Publs Carnegie Inst.; (109):1-735, pl. 1-76.

MLANZAN, H. W. 1986. Estudio sistemático y bioecológico de algunas medusas Scyphozoa de la región subantártica. Tesis doctoral. Universidad Nacional de La Plata, Facultad de Ciencias Naturales y Museo. 196p., 22 lám.

\& SABATINI, M. E. 1985. Estudio preliminar sobre distribución y abundancia de Mnemiopsis maccradyi en el estuario de Bahía Blanca. Spheniscus, 1:53-68.

MÖLLER, H. 1980. Population dynamics of Aurelia aurita medusae in Kiel Bight, Germany (FRG). Mar. Biol., 60:123-128.

RUSSELL, F. 1970. The medusae of the British Isles. II. London, Cambridge University Press. 284p.

VALENTINUZZI de SANTOS, S. 1971. Estudio preliminar sobre las comunidades intercotidales del Puerto de Ingeniero White (Provincia de Buenos Aires). Physis, Sec. A, 30 (81):407-416. 
VANNUCCI, M. \& TUNDISI, J. 1962. Las medusas existentes en los museos de La Plata y Buenos Aires. Comun. Mus. argent. Cienc. nat. Bernardino Rivadavia, Cienc. zool., 3(8):203-215.

(Recebido em 06-10-87; aceito em 16-10-89) 